\title{
Ueber die Bildung der Trennungslinien in den Saturnsringen.
}

Von Dr. M. Wilhelm Meyer.

Schon im Jahre 1868 hatte Kirkwood darauf hingewiesen, dass ein Saturnsatellit, welcher sich in der Entfernung der Cassini'schen Trennung um den Planeten bewegen wüde, eine Umlaufszeit besitzen müsste, die nahezu com. mensurabel mit denen der vier inneren Satelliten des Systems wäre. Es ist bekannt, dass bei einer solchen Commensurabilität der Umlaufszeiten zweier Körper desselben Systems deren gegenseitige Störungen sich stets in demselben Sinne summiren, bis beide Bahnen einen vollständig verschiedenen Character angenommen haben. Unter der Voraussetzung, dass die Saturnsringe aus einer grossen Zahl .sich selbstständig bewegender Körper bestehen, erklärt sich also die
Bildung der grossen Trennungslinie in den Ringen durch den störenden Einfluss der Satelliten, welcher in dieser Entfernung vom Saturn ein Maximum ist. Ich habe nun diese Frage etwas weiter verfolgt, und alle möglichen Combinationen von Commensurabilitäten der Umlaufszeiten aufgesucht, welche für mehrere Satelliten zugleich in derselben Entfernung vom Saturn störend wirken müssen. Auf diese Art entstand folgende Tafel, welche sämmtliche bekannten Trennungs- beziehungsweise Grenzlinien des Systems bis auf sehr geringe Differenzen mit der Beobachtung übereinstimmend durch Rechnung wiedergiebt.

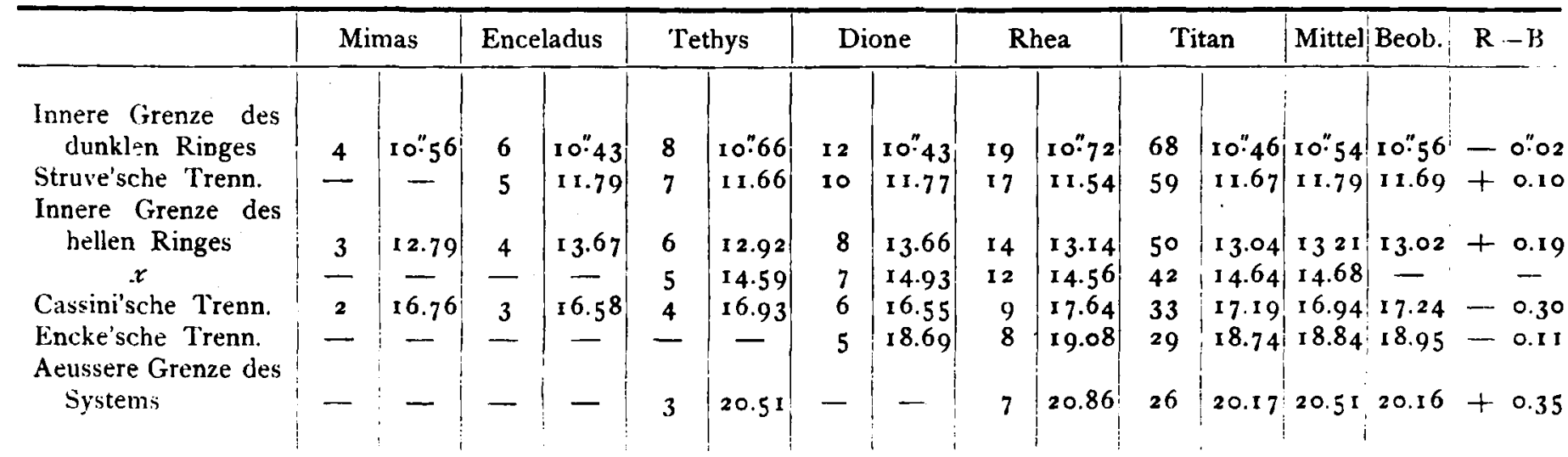

In dieser Tabelle giebt die erste Reihe für jeden satelliten die ganze Zahl, welche die betreffende Commensurabilität der Umlaufszeiten characterisirt, während die zweite Reihe die Entfernung vom Saturnmittelpunkte angiebt, in welcher zufolge dieser Commensurabilität die Störungen des betreffenden Satelliten maximale sind.*) Die Reihe $>$ Mittel \% enthält das einfache Mittel der in derselben Horizontalreihe angefürten Distanzen; die folgende Reihe enthält die beobachtete Distanz für die betreffende Parthie der Ringe und die letzte schliesslich den Unterschied der beiden vorhergehenden Zahlen.

Die nähere Betrachtung dieser Zahlenreihen lässt aus der Rechnung noch manche Eigenthümlichkeiten der Configuration der Ringe erkennen, welche die oben entwickelten Voraussetzungen und Schlüsse frappant bestätigen. Die erste Zahlenreihe, welche sich auf die innere Begrenzung des dunklen Ringes bezieht, giebt alle sechs Entfernungen für die Einflüsse der inneren Satelliten sehr nahe übereinstimmend an. Dieser Umstand erklärt die durchaus scharfe Begrenzung des dunklen Ringes nach seiner inneren Seite hin, welche allen Beobachtern aufgefallen ist. Alle sechs Satelliten vereinigen hier ihre störenden Einflüsse um einzelne Partikeln, welche diese innere Grenze zeitweise überschreiten, zu sich nach aussen heranzuziehen. Die Uebereinstimmung

*) Als Einheit ist in die Rechnung die halbe grosse Achse der Titansbahn, wie sie aus meiner später mitzutheikenden Bahnrechnung Yolgt, eing eführt. der durch die Rechnung gefundenen Mittelzahl mit meinen Beobachtungen ist vollkommen.

Die Struve'sche Trennungslinie in dem dunklen Ringe wird durch die Störungen der Satelliten von Enceladus an erzeugt, und zwar ist die Coïncidenz der fünf oben mitgetheilten Zahlen noch vollkommener als in der vorhin betrachteten Zahlenreihe. In der Rubrik Beob. \& habe ich hier eine Distanz angegeben, welche in der Mitte zwischen der inneren Begrenzung des dunkeln und des hellen Ringes liegt. Die Zahl kann nach den Angaben der wenigen Beobachter dieser Linie von ihrer wirklichen Entfernung nicht wesentlich abweichen. Ich habe die Linie nie gesehen. Ihre Existenz ist bekanntlich öfters angezweifelt und O. Struve selber hat sie 1882 nicht wiedersehen können, während Schiaparelli sie deutlich sah. Die Rechnung beweist die Nothwendigkeit dieser Trennungslinie.

Die innere Grenze der hellen Ringe ist eine besonders deutlich markirte Stelle im System. Hier vereinigen sich in der That die Einflusse aller in Rechnung gezogenen Satelliten, um diese Configuration zu bilden. Jedoch sind die Coïncidenzen der einzelnen Zahlen viel unvollkommener, wie zum Beispiel für die innere Begrenzung des dunkeln Ringes; es existirt hier eine Region von beinahe einer Secunde Ausdehnung, in welcher die störenden Einflüsse sich vermischen. Aus diesem Umstande erklärt sich der sehr allmälige Uebergang des inneren hellen Ringes in den dunklen, so dass diese innere Grenzlinie stets verwaschen und undeutlich erscheint. 
Anders verhält es sich mit der Cassini'schen Trennung. Diese auffälligste Stelle des Systems wird gleichfalls durch die störenden Einflüsse sämntlicher Satelliten erzeugt, und zwar erkennt man sofort, dass diese hier grösser sein müssen, als auf irgend einem anderen Gebiete des Ringsystems, weil für die beiden innersten, also am stärksten wirkenden Satelliten Mimas und Enceladus die betreffenden Commensurabilitäten durch kleinstınögliche Zahlen repräsentirt, also möglichst vollkommen sind. Die Commensurabilität 2 für Enceladus würde ausserhalb des Systems der Ringe fallen. Für die vier nächsten Satelliten fallen die betreffenden Distanzen nahe zusammen, und zwar coïncidiren sie mit der inneren Begrenzungslinie der Trennung, welche beinahe eine halbe Secunde breit ist. In diesern Zusammenwirken der vier inneren Satelliten ist der Grund zu suchen, weshalb die innere Begrenzung der Trennung besonders scharf markirt ist. Die äussere Begrenzung, also der Beginn des äusseren hellen Ringes ist viel undeutlicher. Er scheint von innen her ganz wie der innere Ring mit äusserst schmalem Profil zu beginnen, um sich erst ganz allmälig in etwas grösserer Entfernung etwas mehr auszudehnen. Die Ursache hiervon liegt in dem schwächeren und in grösserer Entfernung vom Saturnmittelpunkte wirkenden Einflusse von Rhea und Titan.

Was nun die Encke'sche Trennung betrifft, so wird dieselbe nur durch drei Satelliten erzeugt, von denen Dione den bei Weitem vorwiegenden Einfluss hat. Die Feinheit und Schwäche dieser Linie ist dadurch erklärt. In der Rubrik Beob. habe ich eine Distanz angenommen, welche aus den früher mitgetheilten Beobachtungen über die relative Lage dieser 'Trennung als Mittelstellung folgt.

Die äussere Begrenzung des Systems scheint hauptsächlich durch Tethys, welche hier den stärkstmöglichen Einfluss ausübt, entstanden zu sein. Die Einflisse von
Rhea und besonders Titan muss man meiner Ansicht nach nur sehr gering anschlagen, weil ihre Commensurabilitäten stets durch grosse Zahlen repräsentirt sind und namentlich diejenigen Commensurabilitäten, welche in der vorhergehenden Tabelle für diese Satelliten nicht verwendet sind, also mit denen der inneren Satelliten nicht coüncidiren, im Ring. systeme keinen merklichen Einfluss ausgeübt haben.

Während nun alle möglichen Commensurabilitäten der beiden innersten Satelliten Mimas und Enceladus, welche auf Regionen innerhalb des Ringsystems störend wirken, ihre sichtbaren Spuren in demselben markirt haben, bleibt für Tethys nur noch die Commensurabilität 5 unverwendet, welche in derselben Region zusammenwirken muss mit der Commensurabilität 7 für Dione, 12 für Rhea und 42 für Titan. Ein Vergleich mit den betreffenden Zahlen, welche für die Encke'sche Trennung gelten, zeigt sofort, dass der Einfluss der soeben genannten Satelliten noch ein wenig stärker sein muss als der, welcher die Encke'sche Trennung erzeugt. Sind nun die oben entwickelten Schlussfolgerungen richtig und die bekannten Trennungslinien im Saturnsystem durch die Störungen der Satelliten erzeugt, so muss sich mit den uns gegenwärtig zu Gebote stehenden optischen Mitteln in der Entfernung 14.7 vom Saturnmittelpunkt eine schwache und feine Trennung entdecken lassen, welche den inneren hellen Ring ungefiihr in einer Entfernung von zwei Dritteln der ganzen Breite desselben von der Cassini'schen Trennung nach innen gerechnet theilt. Es wäre also wünschenswertis, dass die Astronomen dieser Stelle ihre besondere Aufmerk. samkeit zuwenden möchten.

Genf 1883 Juli 10.

\section{Notiz uiber einige neue und ältere Nebel.}

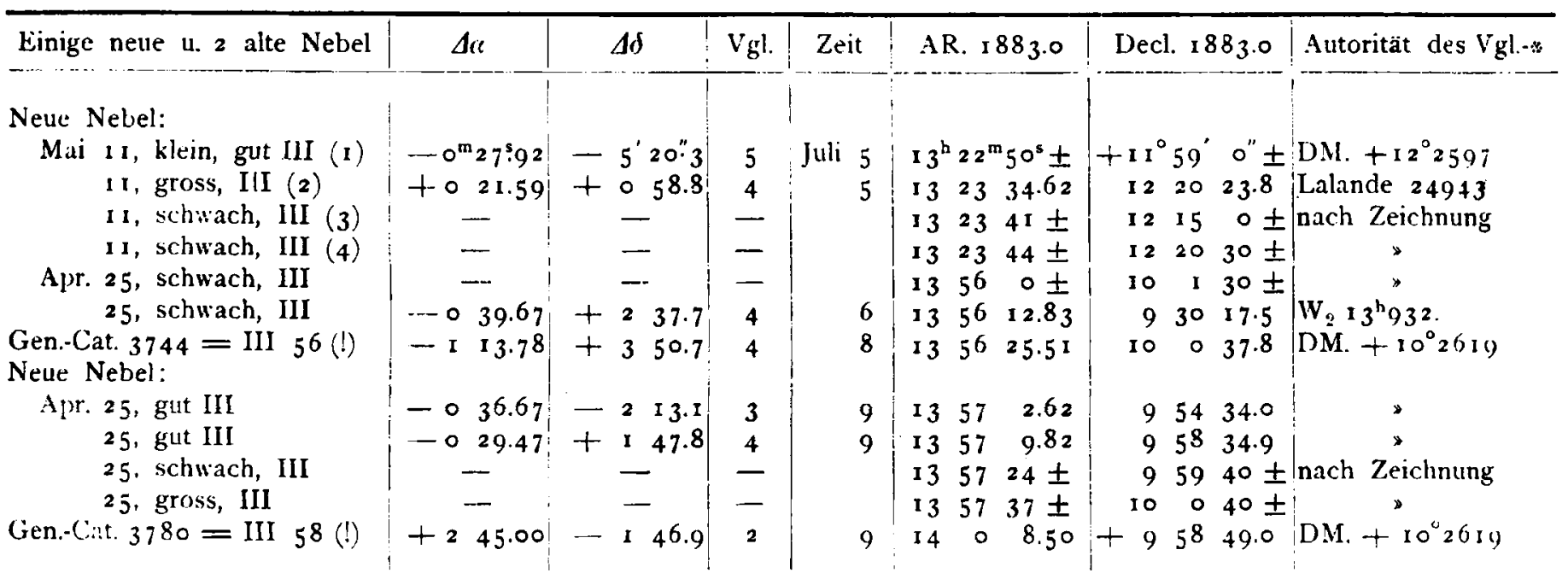

(Ueber die Angabe: snach Zeichnung erwähne ich nur, dass ich bekannte Sterne in der Nähe reducirte und den nicht messbaren Nebel auf einer Zeichnung im grossen Maassstibe eintrug. Ich glaube, der Fehler wird nicht $1 / 2$ übersteigen.) 